\title{
Repensar la protección social en salud en América Latina y el Caribe ${ }^{1}$
}

\author{
Hernán Rosenberg ${ }^{2}$ y Bernt Andersson ${ }^{2}$
}

RESUMEN A pesar de lo que señalan las Constituciones y otras normas básicas de los países de la Región de las Américas, la exclusión de la protección social en salud es un problema que afecta a una proporción importante de la población (al menos 20\% y, en números absolutos, entre 80 y 200 millones de personas). A esta cifra se llega a través de una serie de indicadores teóricos (cober tura de la seguridad social) y prácticos, tanto de estructura (pobreza, etnia y barreras geográfi cas) como de proceso de salud (partos que tienen lugar fuera de instituciones de salud, cumpli miento del calendario vacunal y acceso a saneamiento básico). La exclusión se ve afectada por el nivel de segmentación de los distintos subsistemas de salud de una sociedad. Tradicionalmente existían en la Región de las Américas los subsistemas público, privado y de seguridad social. La falta de respuesta a este problema ha motivado el surgimiento de un subsistema comunitario. La coexistencia de distintos sistemas combinada con la falta de regulación por las autoridades sani tarias ha ocasionado altos niveles de exclusión y una alta ineficiencia en el uso de los recursos del sector. En el contexto de la reforma del sector de la salud de cada país, se recomienda un pro ceso de diálogo social en el que se discuta explícitamente el tema de la protección social en salud. Este proceso, que debe ser organizado de forma participativa, debería incluir un diagnóstico com pleto de la situación (cuántos son los excluidos, quiénes y por qué, y cuáles son los mecanismos más adecuados en el contexto nacional para combatir el problema) y una evaluación técnicopolítica de las opciones más convenientes para cada sociedad, en la que se determine si se han agotado o no las posibilidades de los subsistemas tradicionales. El proceso debería culminar con un programa de implementación de las propuestas en el que tengan cabida las distintas modali dades más adecuadas para cada caso, de forma que se logre la máxima protección social en salud.

El concepto de protección social en salud (PSS) ha variado en el tiempo. Originalmente comunitaria, la PSS evolucionó hasta llegar en las Américas a una propuesta en la cual el Estado aseguraba la atención a través de la provisión de servicios. En la acepción más moderna, sin embargo, la responsabilidad social

\footnotetext{
Este artículo se basa en las discusiones y documentos de la Reunión Regional Tripartita de la Organización Internacional del Trabajo con la colaboración de la Organización Panamericana de la Salud sobre "Extensión de la protección social en salud a los grupos excluidos en América Latina y el Caribe", que se efectuó en Ciudad de México del 29 de noviembre al 1 de diciembre de 1999, en el contexto del programa de colaboracion entre ambas organizaciones sobre la "Extensión de la Protección Social en Salud". Los
}

consiste en conseguir que la población tenga acceso a la protección y al cuidado de su salud a través de la asignación a ese fin de recursos de distinto origen. La provisión de los servicios, e incluso la gestión de los recursos mismos, puede ser una responsabilidad individual, una responsabilidad social o, lo que es más

autores fueron miembros del secretariado de la reunión.

2 División de Desarrollo de Sistemas y Servicios de Salud, Organización Panamericana de la Salud/Organización Mundial de la Salud, Washington D.C. Toda la correspondencia debe ser enviada a Hernán Rosenberg a la siguiente dirección postal: HSP, PAHO/OPS, 525, Twenty-third Street NW, Washington, D.C. 20037, Estados Unidos de América.

3 Para estos efectos estamos usando la definición de salud de la Organización Panamericana de la frecuente, una responsabilidad compartida. La PSS pasa a ser entonces la garantía que los poderes públicos otorgan para que un individuo o grupo de individuos puedan obtener servicios de salud de una manera adecuada a través de alguno o algunos de los subsistemas de salud existentes en el país. 3,4

Salud/Organización Mundial de la Salud, como el estado de bienestar físico, psíquico y social del individuo.

4 Panorama de la exclusión de la protección social en salud en América Latina y el Caribe. Informe de la Organización Internacional del Trabajo/ Organización Panamericana de la Salud presentado a la Reunión Regional Tripartita sobre "Extensión de la protección social en salud a los grupos excluidos en América Latina y el Caribe". México, 29 de noviembre-1 de diciembre de 1999. 
Según las Constituciones o leyes orgánicas de los países de América Latina y el Caribe (ALC), este artículo no debería ser necesario, ya que todos los países han declarado directa o indirectamente que la salud es un derecho de todos sus habitantes. ${ }^{5}$ Sin embargo, una revisión de los datos existentes nos indica que el número de personas sin una efectiva PSS es significativo. Esta población se llama excluida (1) y el fenómeno exclusión.

En este artículo revisaremos, en primer lugar, las dimensiones de la exclusión y las características de los principales afectados. Enseguida analizaremos las estructuras de los sistemas de salud y sus características e implicaciones para la exclusión. Finalmente, estableceremos un marco desde el cual se pueda articular un programa para su disminución.

\section{LA EXCLUSIÓN}

La magnitud de la exclusión en ALC es considerable. Su naturaleza es multicausal y para analizarla se requiere la utilización de diversas ópticas; es decir, no existe una única manera de analizar los datos relevantes. Es importante notar que, si bien la exclusión y la pobreza están asociadas, no son sinónimos. Hay personas pobres con acceso a los servicios de salud y otras que no son pobres y no tienen acceso a ellos.

\section{Implicaciones de la exclusión}

Podemos analizar las siguientes:

A) Sobre la salud de la población. Si la población no tiene acceso regular a los servicios de salud, naturalmente su salud y bienestar se ven afectados. Por ejemplo, la exclusión de los servicios de vacunación y otras actividades preventivas aumenta el

\footnotetext{
5 Elementos para el análisis comparado de la extensión de la cobertura social en salud en América latina y el Caribe. Informe de la Organización Internacional del Trabajo/Organización Panamericana de la Salud presentado a la Reunión Regional Tripartita sobre "Extensión de la protección social en salud a los grupos excluidos en América Latina y el Caribe". México, 29 de noviembre-1 de diciembre de 1999.
}

riesgo de enfermedades. La exclusión de los servicios curativos implica que las enfermedades pueden agravarse antes de que las personas finalmente consigan llegar a un servicio de salud, con el consiguiente riesgo de muerte o discapacidad.

B) Sobre la equidad. La exclusión es una desigualdad entre las personas que es injusta y evitable. La falta de acceso regular a servicios de salud de una proporción importante de las personas es inaceptable desde el punto de vista ético, además de ser anticonstitucional en todos los países de la Región.

C) Sobre la opinión pública. La progresiva globalización y el mayor acceso a la información hacen de la exclusión un factor de presión social, ya que la población está cada vez más consciente de que personas con nivel socioeconómico similar tienen mejor acceso a los servicios de salud en otros países.

D) Sobre la efectividad y la eficiencia del gasto individual. La falta de PSS ocasiona un gasto en salud exagerado e ineficiente para los excluidos. Ante la perspectiva de tener que pagar de su bolsillo una prestación, la tendencia natural es a postergar el gasto todo lo posible. Esto conlleva gastos inoportunos y a menudo innecesariamente altos, o bien un uso fraudulento de las instalaciones más caras de los servicios de salud, como los servicios de urgencias. $^{6}$ Este fenómeno suele afectar más a la población más pobre. En efecto, las encuestas de hogar han comprobado que las personas de los quintiles inferiores terminan gastando en salud una proporción mucho mayor de su ingreso que las de los quintiles superiores. ${ }^{7}$

\footnotetext{
6 Adicionalmente, la exclusión se puede traducir en el uso de servicios por la vía de la suplantación de personas cuando un individuo excluido utiliza los servicios simulando ser un beneficiario.

7 El gasto de bolsillo en salud en América Latina y el Caribe: razones de eficiencia para la extensión de la protección social en salud. Informe de la Organización Internacional del Trabajo/Organización Panamericana de la Salud presentado a la Reunión Regional Tripartita sobre "Extensión de la protección social en salud a los grupos excluidos en América Latina y el Caribe". México, 29 de noviembre-1 de diciembre de 1999.
}

E) Sobre la eficiencia del sistema en su conjunto. En todo sistema de seguro, mientras mayor sea el tamaño de la población cubierta, mayor es la probabilidad de que más gente sana "compense" el gasto de los enfermos. La exclusión puede afectar entonces a la eficiencia del sistema en su conjunto, por un lado al disminuir la base de reparto de los riesgos y, por otro, al aumentar la probabilidad de ocurrencia de eventos caros.

\section{Dimensiones de la exclusión}

La medición de la exclusión puede hacerse desde un punto de vista interno o externo al sector de la salud, dependiendo de que las estadísticas de base se manejen dentro o fuera del sector. A continuación se tratan ambos tipos de enfoques del tema. ${ }^{8}$

Usando indicadores externos, una primera medida de la exclusión consiste en considerar el número de personas que no están cubiertas por los sistemas de seguridad social (SS). En ALC, la SS cubre a los trabajadores formales que destinan parte de sus ingresos, generalmente cofinanciados por el gobierno o los empleadores, a primas de seguro que cubren tanto la jubilación como las prestaciones de salud. En algunos casos, los familiares dependientes de los asegurados también están cubiertos. Un análisis de las legislaciones sobre la SS indica que los niveles teóricos de cobertura de los sistemas de SS dejan excluidos a unos 218 millones de personas (cerca de $40 \%$ de la población). Obviamente, parte de la población excluida por este criterio puede tener otros tipos de cobertura, ya sea del sector público o del sector privado.

Una segunda medida se basa en medir ciertas disparidades de acceso. Aquí consideraremos el nivel de po-

\footnotetext{
8 Panorama de la exclusión de la protección social en Salud en América Latina y el Caribe. Informe de la Organización Internacional del Trabajo/Organización Panamericana de la Salud presentado a la Reunión Regional Tripartita sobre "Extensión de la protección social en salud a los grupos excluidos en América Latina y el Caribe". México, 29 de noviembre-1 de diciembre de 1999.
} 
breza, la restricción geográfica y los antecedentes culturales.

El indicador de pobreza mide el número de personas por debajo de lo que la Comisión Económica para América Latina y el Caribe (CEPAL) considera el ingreso mínimo necesario en un determinado país. La carencia absoluta de recursos origina una falta de poder adquisitivo que impide la satisfacción de necesidades mínimas, entre ellas los cuidados de salud. Es razonable suponer que una importante proporción de los pobres, aunque no todos, forman parte de los excluidos. La estimación es que el número de personas por debajo de la línea de pobreza en ALC es de 121 millones.

La restricción geográfica mide el número de personas que, aunque tienen derecho a acceder a los servicios de salud, en la práctica están demasiado aisladas o sencillamente no tienen servicios en su comunidad. El número de estas personas se ha estimado en 107 millones.

Por último, consideremos la disparidad cultural. Ciertos grupos, principalmente por sus características étnicas, no se acercan a los servicios por una percepción de que no serán respetadas sus costumbres, por el riesgo real o supuesto de maltrato o porque prefieren utilizar sistemas tradicionales de medicina o curandería. El nivel de exclusión por este concepto no se ha logrado medir con precisión, pero se estima en unos 43 millones de personas (2). Además, también hay datos que señalan la existencia de un problema de género (3).

Estos criterios no son mutuamente excluyentes, pero tampoco son idénticos. Por ejemplo, si bien una cantidad importante de etnias indígenas se encuentran en los quintiles inferiores de ingreso y viven en zonas no bien servidas, esto no es cierto para todos sus miembros. Algunos pertenecen a grupos con mayores ingresos o viven en zonas con acceso razonable a los servicios de salud. Por este motivo, es necesario conocer en detalle cada situación particular antes de combinar las distintas medidas de exclusión presentadas.
La exclusión también se puede estimar mediante indicadores internos del sector, ligados al proceso de atención sanitaria. Así, se puede reconocer la existencia de prestaciones aceptadas por la comunidad como "necesarias" y "útiles", en el sentido de que nadie las evitaría si pudiera (por ejemplo, las prestaciones de salud maternoinfantiles). Su no utilización indicaría, por lo tanto, una verdadera falta de acceso.

Como primer indicador de este tipo consideremos el número de partos no asistidos por personal entrenado. El registro de los nacimientos se obtiene de los registros civiles, y el número de partos que tienen lugar en instituciones, de las estadísticas de salud. La diferencia es el número de nacimientos no asistidos. Si se corrige por la tasa de fecundidad y el tamaño medio de las familias, es posible calcular el número de personas excluidas de esta prestación, que en ALC asciende a 83 millones.

Otro indicador de este tipo es el cumplimiento del calendario vacunal. Es razonable suponer que si no se cumplen estos programas es básicamente porque las familias consideran que hacerlo no compensa los costos (de transporte, de oportunidad u otros), o porque no están en condiciones de hacerlo. Suponiendo que nadie desea desproteger a sus hijos, ambas explicaciones sugieren exclusión. Utilizando cálculos similares a los anteriores, en ALC se obtiene un número aproximado de 82 millones de personas excluidas.

Por último, se puede considerar el porcentaje de población sin acceso al agua potable y alcantarillado, indicadores relevantes para medir el acceso al saneamiento básico. Por esta vía llegamos a unos 152 millones de personas excluidas.

En resumen, podemos concluir que, cualquiera que sea la medida utilizada, estamos hablando de más de $20 \%$ de la población de ALC. Cuál es el indicador que mejor refleja la realidad depende de las condiciones particulares de cada país. Por ejemplo, la mayoría de los países del Caribe de habla inglesa tienen sistemas nacionales de aseguramiento en salud, por lo que sus sistemas de SS se limitan a cubrir el desempleo y las pensiones, pero no la salud. Por este motivo, el indicador de falta de cobertura de la SS no resulta adecuado en estos países. El indicador cultural es muy relevante en países con diferentes grupos étnicos importantes, como Bolivia, Guatemala, Perú o Ecuador. Sin embargo, puede no ser un buen reflejo de la situación en países más homogéneos, como Uruguay. Por esta razón, en este ensayo se presenta la serie completa de indicadores en el nivel continental.

Aunque aquí nos referimos básicamente a los aspectos cuantitativos de la exclusión, medidos en términos de acceso y uso de los servicios, vale la pena notar que la insuficiente oportunidad o calidad de los servicios puede ser también un factor de exclusión. No obstante, la medición de la calidad de la atención es un tema para investigaciones futuras. Baste decir aquí que los números presentados, a pesar de sus dimensiones, todavía subestiman el problema o nos dan su cota inferior.

A pesar de que la identificación precisa de los excluidos es incipiente aún, ya se han identificado algunos grupos (4). Los pobres en general, las mujeres, los niños, los adultos mayores, los grupos étnicos indígenas, los trabajadores temporales o informales y los desempleados y/o subempleados constituyen los grupos con mayor riesgo de exclusión. Además, la población rural y dispersa tiene menos probabilidades de acceder a la cobertura de los servicios de salud.

\section{LA ESTRUCTURA DE LOS SISTEMAS Y SUS IMPLICACIONES EN LA EXCLUSIÓN}

Las funciones básicas que se ejercen en los sistemas de salud son: a) la rectoría (conducción, regulación y vigilancia); b) el financiamiento (recolección, gestión y asignación de los recursos financieros); c) el aseguramiento (definición de las poblaciones cubiertas y de las prestaciones ofrecidas, así como la compra de los servi- 
cios necesarios para atenderlas), y d) la provisión de servicios (atención a los usuarios de los servicios de salud, razón de ser última de los sistemas de salud). A menudo, los mecanismos de pagoa los proveedores de servicios establecen el vínculo entre las funciones de aseguramiento y provisión.

Estas funciones se ejercen a través de diversos organismos, instituciones y redes de agentes que suelen agruparse en varios subsistemas que establecen relaciones estructurales entre sí (5-7). Los principales se describen a continuación, con énfasis en sus relaciones con el fenómeno de la exclusión.

La seguridad social. Este subsistema fue pensado desde su origen como una manera de proteger al trabajador, principalmente al que tiene empleo formal. Suele contemplar uno o más sistemas de prestaciones asociado con una prima pagada por el trabajador, generalmente complementada por su empleador o el Estado. Estas instituciones tienden a restringir la provisión de servicios a sus asegurados, entre los que últimamente se están incluyendo los familiares dependientes de los trabajadores. Entre los excluidos de este subsistema se encuentran los trabajadores del sector informal, los estratos más pobres y las mujeres jefas de hogar.

La asistencia pública. Este subsistema es financiado a través de los impuestos, generales o locales. En principio cubre a toda la población y en general tiende a prestar servicios mediante trabajadores de la salud que son funcionarios. Aunque fue diseñado para brindar atención gratuita, existe una tendencia reciente a la recuperación de costos mediante mecanismos de copago. En este subsistema, la exclusión depende en buena medida del grado de segmentación de las redes de prestadores. En países con redes únicas o integradas, como Costa Rica, Cuba o los países del Caribe de habla inglesa, la mayoría de la población está cubierta y la exclusión es baja. En aquellos donde la segmentación es muy alta, la exclusión del sistema público obedece a su falta de recursos o a las ineficiencias de asignación y uso de los mismos. A medida que aumenta la integración aumenta la población cubierta y disminuye la exclusión.

En el subsistema privado la exclusión suele provenir del costo de la prima o de los servicios, de las condiciones para ser admitido (por ejemplo, edad, estado de salud previo, períodos de carencia y otros), de la ocurrencia de determinadas situaciones durante el período de vigencia del seguro (por ejemplo, situaciones no cubiertas y riesgos catastróficos) o de una combinación de todos estos factores.

A raíz de las condiciones de exclusión, algunos grupos o comunidades sin acceso a los servicios de salud se han organizado en subsistemas comu nitarios que tienden a ser manejados como seguros. Sobre esta base, compran servicios a proveedores tanto públicos como privados. Estos mecanismos tienden a responder a situaciones concretas de exclusión. Su factibilidad financiera es frágil, particularmente cuando se incluyen enfermedades catastróficas o de alto costo (8).

Lo normal en ALC ha sido la coexistencia de todos estos subsistemas, a menudo sin adecuada regulación y sin una clara dirección. Es importante notar que la coexistencia no implica necesariamente competencia entre sistemas desde el punto de vista del usuario, que generalmente tiene pocas posibilidades de elección si es miembro de alguna variante de la SS. De no ser así, los que pueden pagar utilizan los subsistemas privados y los demás utilizan los subsistemas públicos o comunitarios, o bien quedan totalmente excluidos.

Una primera conclusión acerca de la exclusión es que se pierden economías de escala por la proliferación y la segmentación de subsistemas. En segundo lugar, tradicionalmente ha existido una marcada tendencia a la integración vertical en los tres primeros subsistemas. Todos tienden a cumplir las tres funciones mencionadas de financiamiento, aseguramiento y provisión de servicios, pero sin relacionarse entre sí. Tanto el subsistema de SS como el privado pueden tener más de un participante. Por supuesto, puede haber compra de servicios entre los subsistemas.
La función rectora solo recientemente está siendo asumida por el sector público. El financiamiento puede hacerse a través de impuestos generales (sector público), específicos del trabajo (SS) o por gastos de bolsillo (privado y copagos de los otros sistemas). El sector privado puede incluir o no un sistema de seguros, pero en ambos casos se paga por el gasto de bolsillo. El sector no convencional se financia por el gasto de bolsillo y/o impuestos a través de subvenciones de los distintos niveles de autoridad pública.

Naturalmente hay excepciones a la coexistencia de todos los subsectores. En Costa Rica, por ejemplo, hay un sistema integrado en el que el Ministerio de Salud asume la rectoría y parte del financiamiento, mientras que la SS ejerce tanto la función de aseguramiento como la prestación. El subsistema privado está separado y tiene un papel menor en este país, pero vende algunos servicios al subsistema público.

En Chile coexisten dos subsistemas, uno público, el Fondo Nacional de Salud (FONASA), y otro privado, las Instituciones de Salud Previsional (ISAPRE), que tienen una rectoría común, aunque la regulación cubre más bien el aseguramiento que la provisión de servicios. Hay compra de servicios entre subsistemas y el subsistema ISAPRE tiene diversos aseguradores que compiten entre sí.

En Cuba y en el Caribe de habla inglesa, la atención tiene lugar a través de un sistema único nacional que cubre financiamiento y prestación. Existen diversos niveles de rectoría. La función aseguradora no se identifica como tal, pero sí se asignan o distribuyen los recursos presupuestados a través de los organismos públicos.

El gasto en salud per cápita suele ser muy alto en el subsistema privado, alto en la SS y mediano o bajo en la asistencia pública. La calidad percibida también suele decrecer en el mismo sentido. Desde el punto de vista de la exclusión, el resultado de esta segmentación es regresivo: los que pueden pagarlo utilizan el subsistema privado por su mejor calidad. Los cubiertos por la SS usan estos servicios, pero son un 
número limitado. En consecuencia, los grupos menos protegidos usan el subsistema público, aumentando la carga sobre el mismo y recibiendo el servicio de menor calidad percibida (9).

\section{Exclusión y reforma del sector de la salud}

La mayor parte de los países de ALC han pasado o están pasando por importantes procesos de reforma del sector de la salud $(10,11)$. Es importante notar que la lucha contra la exclusión de la PSS no ha sido un propósito explícito de estas reformas. De hecho,en muchos países las reformas han sido derivadas de procesos de transformación del Estado o de ajustes macroeconómicos. En consecuencia, ha existido una fuerte presión sobre el sector de la salud para mejorar la eficiencia y reducir costos. En muchos casos ello se ha hecho a expensas de la calidad de los servicios. Desde el punto de vista de la exclusión, ambas presiones son regresivas.

Ya se señaló que la instauración de copagos actúa como inhibidor del acceso para las personas de menores recursos relativos. Pero, a pesar de los elevados costos de bolsillo, los excluidos terminan utilizando servicios privados porque no confían en la accesibilidad o capacidad resolutiva de los públicos. Otra consecuencia de las reformas ha sido que los fármacos, que solían ser parte de la atención pública, ahora tienden a no estar cubiertos. Por tanto, los gastos efectivos de bolsillo para los sectores de menores ingresos pueden terminar siendo mayores que los derivados de acudir a un servicio privado.

Las reformas han tendido a impulsar la separación de funciones. Se propugna que el financiamiento, el aseguramiento y la provisión se separen como una manera de mejorar la eficiencia. Más aún, en algunos países se contempla la existencia de uno o más participantes en cada función. Por ejemplo, en Chile se puede elegir entre seguro público y privado, y, dentro del privado, entre varios aseguradores.

En teoría, en términos de eficiencia es posible imaginar un equilibrio óp- timo entre la competencia y la colaboración entre los distintos agentes de un sistema. Las ventajas de la competencia son la mejor atención y los menores costos; una desventaja es que, en determinadas condiciones, se pierden las economías de escala y los costos transaccionales se vuelven prohibitivos; otra es la tendencia a la inequidad por la vía de la selección adversa de riesgos. Por su parte, la mayor colaboración permite una mejor asignación de recursos en el sistema como un todo y tiende a frenar la selección adversa; su desventaja es la tendencia a la ineficiencia, como ocurrió en la mayoría de los sistemas antes de las reformas.

Lo anterior tiene que ver con la dimensión "horizontal", es decir en una misma función. Pero existe también un nivel de colaboración o de competencia "vertical", entre funciones. Los sistemas integrados verticalmente pueden tener un importante nivel de economía de escala, pero menor incentivo a la eficacia y eficiencia. Por ejemplo, en los Estados Unidos de América, hasta hace unos 20 años, la radical separación entre las funciones de aseguramiento y de prestación, unida a la preponderancia del pago de bolsillo por acto médico y a otros factores, generó una escalada de costos que lo hizo casi impagable. De ahí el cambio de las últimas dos décadas hacia modalidades de atención gerenciada que ha resultado en una contención de costos a expensas de la libertad de elección de los usuarios y de los médicos $\mathrm{y}$, en ocasiones, de la calidad percibida del servicio.

La determinación del nivel óptimo de integración vertical $u$ horizontal es una de las decisiones más importantes a tomar en un país, pero es imposible decidir si no se cuenta con una capacidad real de rectoría que permita no solo diseñar los modelos, sino también aplicarlos.

La descentralización y la definición de conjuntos básicos de prestaciones para la población de menores ingresos han contribuido a reducir la exclusión. Otro mecanismo ha sido la introducción o la extensión de seguros sociales de salud, seguros básicos, seguros adicionales o seguros de enfermedades catastróficas y la promoción del acceso universal a estos seguros. Se han desarrollado, además, programas focalizados, dirigidos a los grupos vulnerables, para complementar los programas uni-

\section{Algunas experiencias con modelos alternativos y complementarios de aseguramiento en ALC}

\section{Ejemplo I: Argentina}

La Obra Social Municipal (OSMU) de Trenque Lauquén, en la Provincia de Buenos Aires, fue fundada en 1992 por la Intendencia de dicha localidad, que se encarga de la administración del sistema. La OSMU proporciona cobertura a la población que no goza de acceso a los programas sociales tradicionales. Su cobertura es de unas 18000 personas, que tienen acceso a los niveles primario y secundario de atención, aunque algunas enfermedades crónicas están excluidas. La OSMU se financia por las contribuciones de sus miembros, los copagos y aportes de la Intendencia y de la provincia. Las personas de recursos limitados pueden hacer sus aportes mediante trabajos comunitarios que son reconocidos a través de la Intendencia. La OSMU tiene su propia producción de medicamentos genéricos.

Ejemplo II: Guatemala

La Coordinadora General de Trabajadores Guatemaltecos administra un sistema de seguro de salud que creó en 1996 para sus miembros y que se financia a través de contribuciones específicas de estos y otros ingresos de la organización. El plan proporciona cobertura básica a los trabajadores informales con mínimo acceso a otros sistemas que trabajan en las zonas urbana y periurbana de la municipalidad de Guatemala. Los beneficios incluyen atención primaria ambulatoria, medicamentos y servicios de laboratorio. Su cobertura es de unos 4750 asociados. 
versales. Si bien hay una tendencia a incorporar a las instituciones de SS al diálogo, en la práctica hay resistencias, con meritorias excepciones, como Costa Rica y Panamá. Por una parte los asegurados temen que sus servicios se deterioren y, por otra parte, se movilizan las esperables resistencias burocráticas institucionales.

\section{Límites de los sistemas convencionales}

La primera forma de responder a la demanda de los excluidos a través de los sistemas convencionales sería aumentar la oferta de servicios públicos. Esto presenta una serie de dificultades: a) reticencia al crecimiento de la función pública de provisión de servicios por las razones ya mencionadas; b) crecimiento de un sistema que tiende a ser vertical y menos sensible a las demandas de los usuarios, y c) dificultad de conciliar esta propuesta con la tendencia a la descentralización de la mayor parte de las reformas del sector de la salud.

Una segunda forma de reducir la exclusión es aumentar la cobertura de seguros de los excluidos. Naturalmente, esto supone que habrá una oferta de servicios y que la principal barrera de acceso es la financiera. Esto es posible hacerlo tanto a través de los seguros privados como de las instituciones de SS, ya que, a efectos del asegurador, la inclusión de los excluidos es de efecto neutro si alguien asume sus costos. Sin embargo, la mayor parte de la población excluida no está cubierta precisamente porque no puede pagar. El financiamiento para esta población tendría que ser sufragado, por lo tanto, por el sector público. Suponiendo una buena gestión, esto es, por supuesto, beneficioso para la función aseguradora, pues el aumento de población sirve para disminuir el riesgo. Naturalmente, esto significa aceptar un aumento del gasto en salud, pero focalizado en los excluidos y, siempre que sea posible, sin aumentar la estructura de atención. Combinado con otros ajustes de eficiencia, es posible incluso que el gasto adicional no sea mucho.
En cualquier caso, las reasignaciones de recursos necesarias no serán instantáneas, lo que nos indica que el problema de la exclusión no se verá sustancialmente mejorado en un período de tiempo más o menos largo.

Una segunda limitación de los sistemas convencionales es su capacidad real de llegar a ciertos estratos de la población o grupos étnicos. El problema de la alienación cultural ya se ha descrito, pero hay que preguntarse si tiene sentido ajustar sistemas para beneficio exclusivo de minorías pequeñas o si no sería mejor mantener sistemas complementarios articulados con los convencionales que permitan una atención culturalmente diversa, pero asegurando niveles de atención comparables a los del resto de la población.

Por estas dos razones, conviene revisar el papel que pueden desempeñar los subsistemas complementarios de aseguramiento. Como se mencionó, estos sistemas se crean de facto en una serie de circunstancias en las que los participantes perciben su exclusión. En ALC hay ejemplos que cubren grupos étnicos, nacionales o pequeñas comunidades. Los grupos se crean espontáneamente, en general configurados como aseguradoras, no proveedoras de servicios. La provisión se hace por compra a los subsistemas convencionales, principalmente los públicos.

Una de las características más importantes de estos subsistemas en ALC es su grado de participación comunitaria, que va desde la definición del paquete de cobertura hasta la vigilancia del abuso. La eficiencia está asociada al grado de participación. La mayor dificultad de este tipo de arreglo es su falta de sostenibilidad financiera. Muchas veces fracasan porque se produce un proceso de selección adversa, en el que los sanos abandonan el sistema, con lo

\footnotetext{
Síntesis y análisis de un conjunto de experiencias de microseguros y otras modalidades de extensión de la protección social en salud en América Latina y el Caribe. Informe de la Organización Internacional del Trabajo/Organización Panamericana de la Salud presentado a la Reunión Regional Tripartita sobre "Extensión de la protección social en salud a los grupos excluidos en América Latina y el Caribe". México, 29 de noviembre-1 de diciembre de 1999
}

que aumenta el gasto por participante $\mathrm{y}$, por consiguiente, se deben aumentar las primas o reducir los beneficios. Ello se relaciona con la aparición de enfermedades crónicas con alto consumo de medicamentos o tratamientos o con la existencia de enfermedades catastróficas como el SIDA. La mejor manera de reducir estos riesgos es mediante el reaseguro con los sistemas convencionales, que aumenta considerablemente la base de reparto del riesgo.

En resumen, los sistemas alternativos se justifican mientras los convencionales no puedan llegar a toda la población, ya sea porque el proceso requiere mucho tiempo y recursos o porque es difícil incorporar plenamente ciertos grupos excluidos. Para funcionar, se deben insertar claramente en el sistema, con amplia articulación desde el punto de vista tanto de la compra de servicios como del reaseguro.

\section{CONCLUSIONES}

A pesar de lo que señalan las Constituciones y otras normas básicas de los países de la Región de las Américas, la exclusión es un problema que afecta a una proporción importante de la población (entre 80 y 200 millones de personas). La situación actual es inaceptable y necesita ser abordada directa y abiertamente para encontrar soluciones.

A pesar de algunas mejoras sustanciales del sector de la salud en la Región, no hay muchas pruebas de que las reformas hayan contribuido a mejorar sustancialmente la eficiencia global, la calidad técnica o la percepción de la calidad por parte de los usuarios. Tampoco parecen haber contribuido a la legitimación social de los proveedores ni a la sostenibilidad financiera o institucional de los programas. Las mejoras en términos de acceso a los servicios básicos de salud parecen muy modestas si se considera el tiempo, la energía y los vastos recursos utilizados. Se requiere entonces examinar mecanismos que sirvan para contrarrestar la exclusión a través de los sistemas existentes o de otros complementarios que representen una alternativa válida. 
Importa insistir en que no se está sugiriendo el reemplazo de los sistemas convencionales por otros alternativos. Los sistemas integrados tienen ventajas, suponiendo que se logren eliminar sus ineficiencias y los problemas de atención. Pero, hasta ahora y a pesar de múltiples intentos, los sistemas convencionales no han sido capaces de resolver la exclusión. Los sistemas alternativos han surgido como reacción desesperada de los excluidos y se debe asegurar su inclusión en el sistema a través de los vínculos de servicios y reaseguro. Teóricamente, parece posible definir un sistema que reduzca la exclusión a través de una combinación adecuada de los distintos subsistemas.

Así, en un extremo podemos considerar un sistema completamente integrado, al estilo caribeño, pero con una autoridad concentrada en velar por la disminución y eventual eliminación de la exclusión. En el otro es posible racionalizar la interacción entre la asistencia pública, las instituciones de SS, el subsistema privado y los subsistemas alternativos de aseguramiento, todos ellos actuando de forma integrada para maximizar la equidad y la eficiencia.

Está claro que la presencia de múltiples agentes requiere una fuerte función rectora. En su ausencia es difícil asegurar que la competencia actúe a favor de los excluidos, bajando los costos y mejorando la calidad. Por otra parte, la efectividad de la competencia depende del tamaño del mercado. Por lo tanto, se requiere una clara elaboración técnica de los tamaños mínimos para las distintas entidades que participen en las distintas funciones. Cuando la competencia no sea factible, se debe entrar en un régimen de monopolio regulado, para evitar abusos. La actuación de las autoridades de salud debe ser regida por criterios de justicia, transparencia y equidad, lo cual no es fácil.

La evolución de sistemas de salud caracterizados por una segmentación no regulada hacia una combinación adecuada de coordinación y regulación que asegure la eliminación eventual de la exclusión no es fácil, pero señala una vía importante para la siguiente generación de reformas del sector de la salud que debe realizarse en el contexto de un amplio consenso nacional sobre el modelo más adecuado para cada país. Está claro que no hay soluciones comunes que satisfagan a todos los países y situaciones. Así, no tiene sentido desmantelar sistemas integrados que funcionan razonablemente, como el de Costa Rica; en países con amplias minorías étnicas hay que considerar el respeto a sus costumbres y culturas; en países con sistemas casi colapsados es posible diseñar sistemas nuevos en el contexto de los nuevos arreglos institucionales.
Lo que a continuación se propone es un proceso que incluya una serie de criterios que cubran los principales factores considerados más arriba. El primer elemento es llevar a la agenda política el tema de la exclusión. Es necesario que el tema se haga explícito y disponga de suficiente apoyo técnico como para afectar a la asignación de recursos. El cambio necesario para afrontar la exclusión solo es concebible en el marco de un debate social en el que se planteen todos los valores importantes para la decisión, incluida la equidad y la eficiencia. En segundo lugar, este amplio debate debería incluir un elemento "desde abajo". Las teorías recientes del desarrollo indican que las soluciones no participativas, por $\mathrm{CO}^{-}$ rrectas que sean técnicamente, tienden a alienar a los grupos excluidos. En tercer lugar, es necesario un diagnóstico de la situación particular de cada país en cuanto a la exclusión. ¿Cuáles son sus dimensiones? ¿Quiénes son los excluidos? ¿Qué políticas se han aplicado y cuáles han sido sus resultados? ¿Cuál es la estructura real del sector? ¿Dónde están las resistencias? ¿Hasta qué punto es posible alterar las fuerzas relativas de las distintas instituciones o reasignar funciones? Solo sobre esta base será posible comparar alternativas, hacer propuestas y movilizar a los países hacia una mayor equidad en la cobertura social de salud.

\section{REFERENCIAS}

1. Kliksberg B, comp. Pobreza, un tema impostergable. Nuevas respuestas a nivel mundial. Caracas: Centro Latinoamericano de Administración para el Desarrollo, Fondo de Cultura Económica, Programa de Naciones Unidas para el Desarrollo; 1993.

2. Organización Panamericana de la Salud. Health of the Indigenous People Initiative progress report. Washington, D.C.: OPS; 1998. (Serie Salud de los Pueblos Indígenas, No. 8)

3. Pittman P. Género y calidad de atención: el caso de hipertensión y diabetes en Avellaneda, Provincia de Buenos Aires, Argentina. Washington, D.C.: Organización Panamericana de la Salud; 1999.

4. Tokman V. La informalidad en los noventa: situación actual y perspectivas. Cuadernos de Políticas Sociales $\mathrm{N}^{\circ}$ 2. Secretaría de Desa- rrollo Social. Presidencia de la Nación. Argentina. 1998.

5. Organización Panamericana de la Salud. La Salud en las Américas. Washington, D.C.: OPS; 1998. (Publicación científica No. 569).

6. Castro Gutiérrez A. Evolución de la protección de la salud en el proceso de reformas políticas e institucionales. En II Seminario: Procesos de Reforma de la Seguridad Social en Materia de Salud. Análisis de los procesos en marcha. Conferencia Interamericana de Seguridad Social. Organización Internacional del Trabajo. 1999. (Serie Estudios, No. 47).

7. Mesa-Lago C. Health care for the poor in Latin America and the Caribbean. Washington, D.C.: Pan American Health Organization, Inter-American Foundation; 1992. (Scientific publication No. 539).
8. Dror D, Jacquier C. El micro-seguro: extensión del seguro de salud a los excluidos. Departamento de Seguridad Social, Organización Internacional del Trabajo; 1999.

9. Miranda Radic E. Cobertura, eficiencia y equidad en el área de salud en América Latina. Santiago de Chile: Centro de Estudios Públicos; 1994. (Serie Políticas Sociales No 5 ).

10. Organización Panamericana de la Salud. Perfiles de sistemas de servicios de salud en los países. Washington, D.C.: Iniciativa de Reforma del Sector de la Salud. 1999.

11. Organización Panamericana de la Salud. Foro subregional andino sobre reforma sectorial en salud. Informe de relatoría. Washington, D.C.: Iniciativa de Reforma del Sector de la Salud. 2000. ( $\left.\mathrm{N}^{\mathrm{o}} 21\right)$ 
ABSTRACT Despite what is written in the constitutions and other basic document mandates of the countries of the Region, exclusion from social protection in health (SPH) affects an important proportion of the population (at least 20\%, which represents, in absolute figures, between 80 and 200 million people). These estimates are obtained through a series of theoretical (social security coverage) and practical indicators that encompass structural indicators (poverty, ethnicity, and geographical barriers) as well as process indicators (non-institutional births, compliance with vaccination schedules, and access to basic sanitation). Exclusion levels in a society are affected by the degree of segmentation of the health system. Traditionally, most countries of the Region have had a public, a social security and a private subsystem in health. Lack of attention to the problem has resulted in the formation of a community-based subsystem. The coexistence of many subsystems, along with poor regulation on the part of health authorities, has resulted in high levels of exclusion and inefficient resource allocation within the sector. The organization of social dialogue processes focusing on SPH within the context of health sector reform initiatives in each country is recommended. The process, which should be participatory, should include a full diagnosis of the situation (how many are excluded, who are they, and why, and what mechanisms are the most appropriate for tackling the issue in each country). It should also provide a political and technical feasibility analysis of the most suitable options for each society, and a determination of whether or not conventional subsystems have exhausted their potential. The process should culminate in a program for implementing the specific proposals made in each society, in an effort to maximize SPH. 